\title{
The Numerical Simulations of Clay-Concrete Interface Tests on the Mesoscopic Level
}

\author{
Jinwen Yang, Jianming Zhao a and Xiaosheng Liu \\ State Key Laboratory of Simulation and Regulation of Water Cycle in River Basin, China Institute of Water Resources and \\ Hydropower Research, 100048 Beijing, China
}

\begin{abstract}
The issue of the interface between soils and structures is an important research area in the high embankment dam. The particle flow code $\left(\mathrm{PFC}^{3 \mathrm{D}}\right)$ that uses the discrete element method, can be used to research various geotechnical engineering problems, including the interface problems. Numerical simulations by PFC $^{3 \mathrm{D}}$ entail the mesoscopic parameters of walls and particles, i.e. the cohesion strength, tensile strength, the wall friction and the interparticle friction that are critical to determine whether the results of the simulations can be consistent with those of tests. On the basis of a large number of numerical simulation tests, this paper tries to reveal the connections between these mesoscopic parameters and macroscopic mechanical properties in the clay-concrete interface tests. Then macroscopic and mesoscopic properties of the interface are analysed by numerical simulations.
\end{abstract}

\section{Introduction}

The high embankment dam is the most common type of dam that is normally constructed of earthfill and/or rockfill. According to the World Register of Dams by ICOLD (2018), high embankment dams account for 78\% of all registered dams. Xiaolangdi Dam at $113 \mathrm{~m}$ and Nuozhadu Dam at $261.5 \mathrm{~m}$ in China and Rogun Dam at $335 \mathrm{~m}$ in Tajikistan are typical examples of high embankment dams. The interaction of the interface between the core wall, the key seepage-proof structure, which is made up of compacted clayey earthfill and the bedrock is essentially critical to be figured out, as it influences the response of the core wall remarkably and play an important role in the design and analysis of high embankment dams.

There are various numerical methods used to research the mechanism and behaviour of the interface between structures and soils. The finite element method is widely used to analyse the characteristics and mechanism of the interface. Clough and Duncan [1] proposed the non-linear elastic model Clough-Duncan model for interfaces according to their direct shear tests. Desai $[2,3]$ issued the disturbed state concept based on cyclic tests, which filled the gap in geotechnical engineering in terms of damage mechanics. The elasto-plasticity damage interface model (EPDI) [4] was established on the basis of substantial tests. The model can effectively and accurately anticipate and analyse the evolutional process of interfaces between structures and gravels or other coarse-grained soils. Although many models have been developed for the description of a gravel-structure interface, there is still no efficacious method for the clay-structure interface.

The particle flow code $\left(\mathrm{PFC}^{3 \mathrm{D}}\right)$ software $[5,9]$ is used here to design a series of the interface tests of mesosimulations to simulate the procedure of the tests of the interface between the core wall and the bedrock and then research the interaction of these two materials in the mesoscopic scale. PFC is developed dependent on the discrete element method and its advantages are that it can simulate the big deformation of soils, that there is no need to draw the computational grids and that it can directly illustrate the process of the interaction of the interface. The particle flow code was used to research different geotechnical problems including shear band, failure mechanism and liquefaction [6-8].

\section{Simulation}

\subsection{Simulation procedure}

The purpose of the simulations is to simulate the test of interface between the clay and the concrete bedrock. The simulation procedures are as follows:

Step1 Preparation: basing on the interface test apparatus, as shown in Figure. 1 that its shape is cylinder, of which the radius is $25 \mathrm{~cm}$ and the height is $22.5 \mathrm{~cm}$, the simulation model can be determined. Then according to the porosity of the clay sample which is 0.40 in physical tests, the porosity in numerical simulations is set as 0.40 correspondingly. Choosing a suitable contact model to mimic the mechanical behaviours of clay is very

*Corresponding author: ${ }^{\text {a }}$ zhaojm@iwhr.com 
important and it would be discussed in section 2.2.1 why the parallel bond contact model was chosen. The particle sizes are between $5.75 \mathrm{~mm}$ and $6.25 \mathrm{~mm}$.

Step2 Parameters calibration: the step plays a significant role in the simulation, which determines whether the numerical simulation results fit the physical test results. Mesoscopic parameters in the numerical simulations have a significant effect on macroscopic mechanical properties. These parameters include cohesion strength and tensile strength in the parallel bond contact model, wall and interparticle friction coefficients, all of which are not negligible (discussed in section 2.2). Other parameters i.e. normal and tangential stiffnesses of the contact model, wall and particles are inconsequential and due to the limited space of the paper, these inconsequential parameters would not be discussed in the paper.

Step3 Model generation and stress loading: Figure.2 shows the simulation model, which has the same size and porosity with the physical test and mesoscopic parameters are inputted in this model. Then the normal stresses are applied (there are four normal stress conditions i.e. $1000 \mathrm{kPa}, 600 \mathrm{kPa}, 300 \mathrm{kPa}$ and $100 \mathrm{kPa}$ ) to the specimen using the servo system.

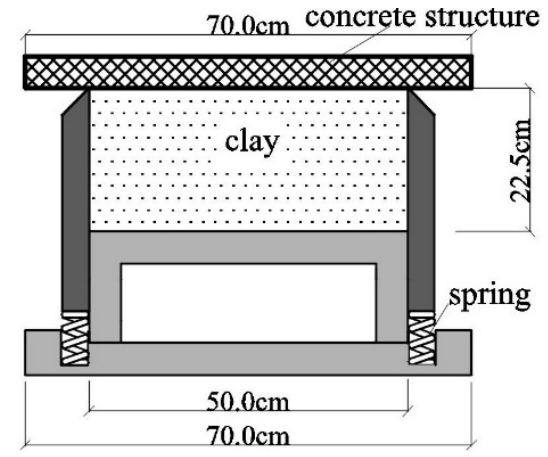

Figure 1. Sketch of interface text apparatus.

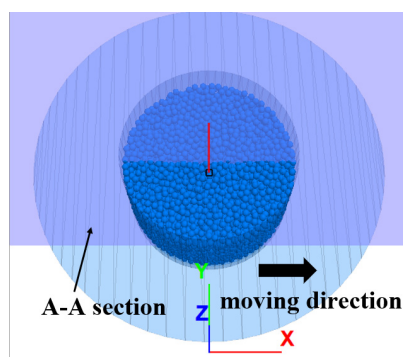

Figure 2. Sketch of the interface text simulation.

Step4 Simulation of interface tests: the top wall move horizontally for $25 \mathrm{~mm}$ and the data are recorded during the process. Then the simulation results are compared with the physical test results; if the results in numerical simulation do not accord well with the physical ones, move back to step2 to determine mesoscopic parameters again until last simulation results are in agreement with physical results.
Step5 Results and analysis: multiply functions in $\mathrm{PFC}^{3 \mathrm{D}}$ can be used to analyse the results.

A-A section in Figure. 2 is a cross section perpendicular to $\mathrm{X}-\mathrm{Y}$ plane and parallel to $\mathrm{X}$-direction and through the center of the interface $(0,0,22.5)$. The cross section is used in section 3.2 for analysis.

\subsection{Calibration of mesoscopic parameters}

The control variable method is adopted to research the effect of mesoscopic parameters on shear stresses. Table. 1 shows all parameters that the simulation needs and parameters in all of these following numerical simulation tests are from it, unless some parameters are expressly stated.

\subsubsection{Cohesion strength and tensile strength of linear parallel bond model}

There are 9 built-in models within PFC, the linear parallel bond model is the optimal one for the simulation of clayconcrete interface tests because it can transmit forces and moments between the particles, and meanwhile does not preclude the possibility of slip [9].

A contact with the linear parallel bond model is active if it is bonded or if the surface gap is less than or equal to zero, as seen in Figure.3. The grid rectangle displays the active state including forces and moments of normal and shear directions.

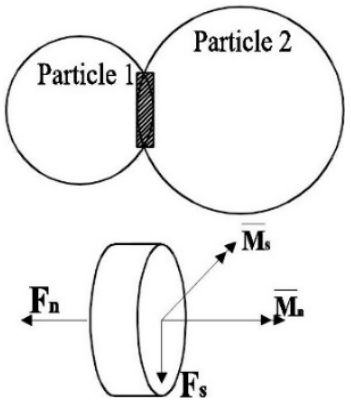

Figure 3. Sketch of the interface text simulation.

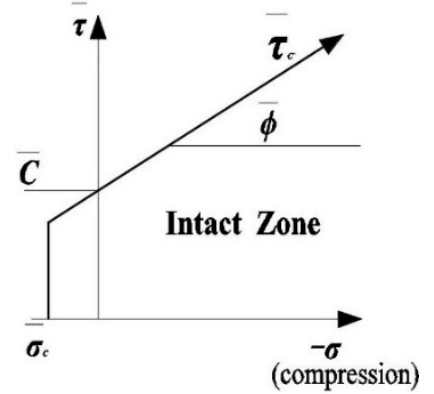

Figure 4. Sketch of the interface text simulation.

As for Figure.4, it illustrates the principle of the breaking bond. If the tensile-strength limit is exceeded, namely, $\bar{\sigma}>\overline{\sigma_{c}}$, the bond in tension is broken; If the bond 
Table 2. Mesoscopic parameters of the numerical simulations.

\begin{tabular}{|c|c|c|c|c|c|c|c|}
\hline \multirow{2}{*}{ Wall } & normal stiffness & tangential stiffness & friction $^{*}$ & \multirow{2}{*}{ Particle } & normal stiffness & tangential stiffness & friction \\
\hline & $6 \mathrm{MN} / \mathrm{m}$ & $6 \mathrm{MN} / \mathrm{m}$ & 0.65 & & $0.6 \mathrm{MN} / \mathrm{m}$ & $0.2 \mathrm{MN} / \mathrm{m}$ & 0.8 \\
\hline \multirow{2}{*}{\multicolumn{2}{|c|}{$\begin{array}{l}\text { Linear parallel } \\
\text { bond model }\end{array}$}} & normal stiffness & \multicolumn{2}{|c|}{ tangential stiffness } & cohesion strength & tensile strength & friction angle \\
\hline & & $800 \mathrm{MN} / \mathrm{m}$ & \multicolumn{2}{|c|}{$800 \mathrm{MN} / \mathrm{m}$} & $3 \mathrm{MPa}$ & $7.5 \mathrm{MPa}$ & $31^{\circ}$ \\
\hline
\end{tabular}

*only the top wall has friction

has not broken in tension, the shear-strength limit is applied to these contacting particles. But if $\bar{\tau}>\overline{\tau_{c}}$, the bond in shear is broken

The most important parameters of the bond model are cohesion strength and tensile strength, which greatly affect the strain softening and hardening, as shown in Figure.5. When cohesion strength is $1 \mathrm{MP}$, there is a phenomenon of obvious strain softening, especially in high normal stress conditions because of the external force mainly coming from the friction force of wall, which splits bonds between particles up and the shear strength has thus been undermined. But when cohesion strength $\geq$ 10MP, such strength makes particles stick tightly together and the external forces are unable to separate one particle from another and this is why the shear stresses in these cohesion strengths are constant (the lower $\sigma$ is, the earlier the shear stress becomes constant) and larger than the values in lower cohesion strengths under all normal stress conditions. When it comes to $0.1 \mathrm{MPa}$ cohesion strength conditions, the curves from numerical simulations tend to show characteristics of gravels somewhat. The adhesion between particles is much looser than the conditions of higher cohesion strength i.e. $1 \mathrm{MPa}$ and $10 \mathrm{MPa}$, of which the initial shear stress paths are completely consistent, and it indicates that the numerical specimen is more like gravel with $0.1 \mathrm{MPa}$ cohesion strength and its shear strength relies on particles themselves instead of the cohesion between particles.

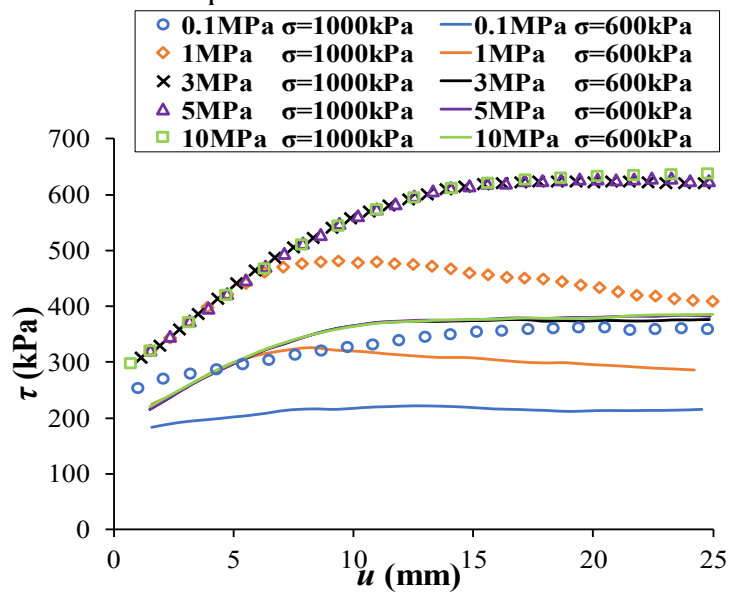

(a) high normal stress conditions

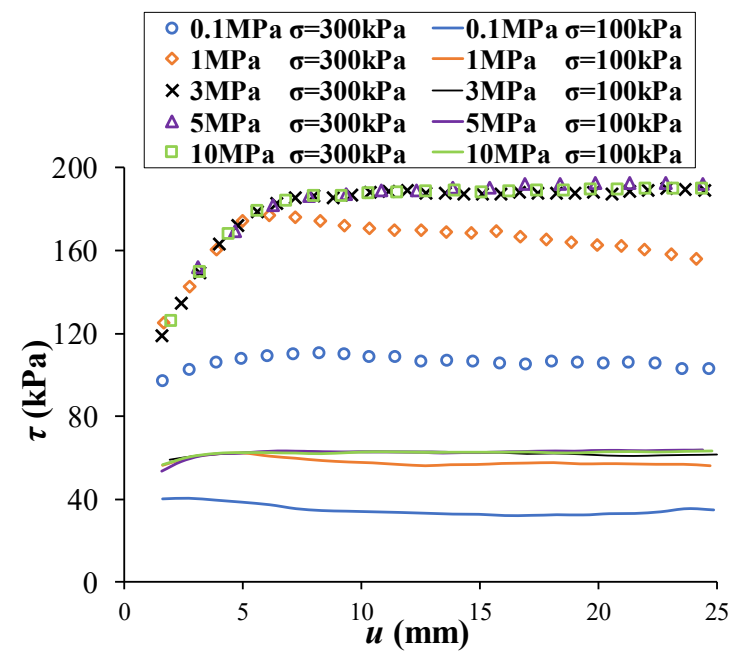

(b) low normal stress conditions

Figure 5. Shear displacement $(u)$ versus shear stress $(\tau)$ with various cohesion strengths.

Table 2. Data of cohesion stresses and peak shear stresses.

\begin{tabular}{|c|c|}
\hline $\begin{array}{c}\text { Cohesion } \\
\text { stress }(\mathrm{kPa})\end{array}$ & $\begin{array}{c}\text { Peak shear } \\
\text { stress }(\mathrm{kPa})\end{array}$ \\
\hline 100 & 361.27 \\
\hline 500 & 391.81 \\
\hline 1000 & 482.01 \\
\hline 3000 & 619.79 \\
\hline 5000 & 629.40 \\
\hline 10000 & 637.12 \\
\hline
\end{tabular}

In order to research $\tau$ - $\sigma$-cohesion relationship, firstly the $1000 \mathrm{kPa}$ normal stress condition is taken as an example to find out a suitable mathmatical function to describe $\tau$-cohesion relationship. By trial and error, the logistic function can be used to fit the data that are shown in Table.2. The following sigmoidal equation (1) is the fitting function for this condition. Figure. 6 displays the data points and fitting curve.

$\tau_{\text {peak }}=636.51-\frac{275.74}{\left(1+\left(\frac{c o h}{1094.44}\right)^{2.65}\right)} \quad\left(\mathrm{R}^{2}=0.9993\right)$

where $\tau_{\text {peak }}$ is the peak shear stress and ' $c o h$ ' is the cohesion strength. 


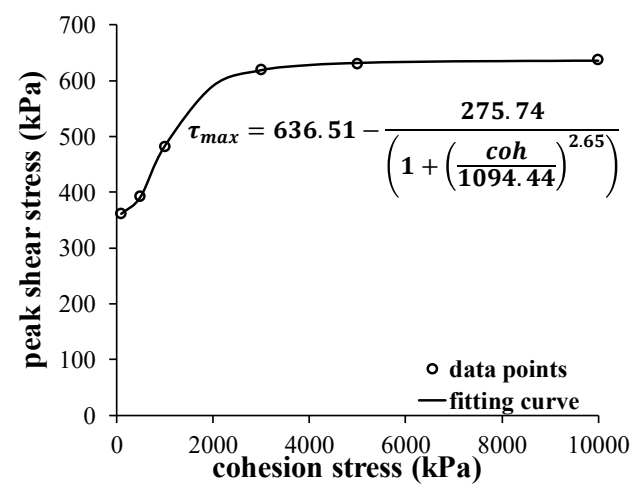

Figure 6. Data points and fitting curve in $1000 \mathrm{kPa}$ normal stress condition.

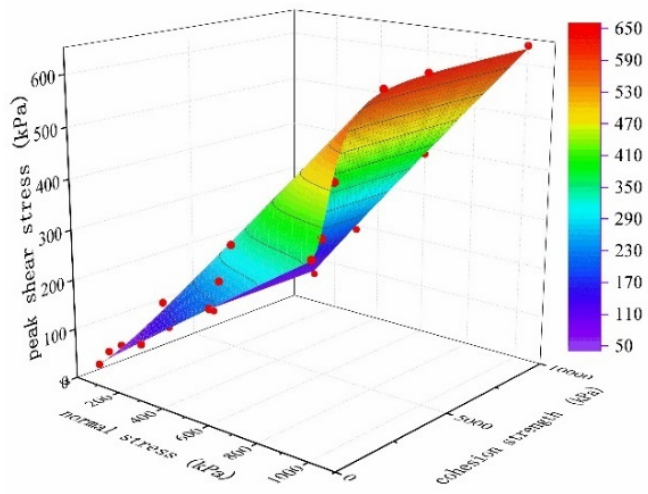

Figure 7. Data points and fitting surface.

All Data of cohesion strength and peak shear stresses in four normal stress conditions are extracted from Figure. 5 and one new variable $\sigma$ is added into the Equation (1) to establish a new function. Considering that there is a positive correlation between normal stress $(\sigma)$ and peak shear stress $\left(\tau_{\text {peak }}\right),\left(\frac{\sigma}{B}\right)^{C}$ (power C here is to modify the correlation because it is not a linear relationship) is used to multiply the logistic function $\left\{A 2-\frac{A 1}{\left(1+\left(\frac{c o h}{D}\right)^{p}\right)}\right\}$. Through trial and error, the following Equation (2) is obtained:

$\tau_{\text {peak }}=3.29+\left\{146.42-\frac{63.65}{\left(1+\left(\frac{c o h}{972.61}\right)^{2.43}\right)}\right\} \times\left(\frac{\sigma}{220.25}\right)^{0.96}$

$\left(\mathrm{R}^{2}=0.9974\right)$

As shown in Figure.7, the peak stresses calculated through Equation (2) are in agreement with numerical simlation results and thus this Equation is competent to describe the $\tau$ - $\sigma$-cohesion relationship, which can be used to predict the peak shear stress or quickly determine the range or even the specific value of cohesion strength in the interfce test.

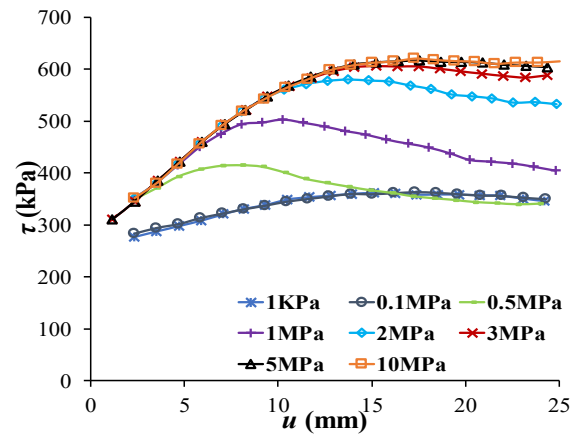

Figure 8. Shear displacement $(u)$ versus shear stress $(\tau)$ with various cohesion strengths in $1000 \mathrm{kPa}$ normal stress.

Tensile strength has a similar trend to cohesion strength. Still taking the $1000 \mathrm{kPa}$ normal stress condition as an example, Figure. 8 shows when the tensile strength exceeds $3 \mathrm{MPa}$, slight increase could be seen as the tensile strength increases between $3 \mathrm{MPa}$ and $10 \mathrm{MPa}$; when the tensile strength $\leq 3 \mathrm{MPa}$, there is an obvious phenomenon of stress softening and softening degree decreases with the decrease of softening stress. Until the tensile strength $\leq$ $0.1 \mathrm{MPa}$, the softening degree becomes insignificant. In these simulations, tensile strength is set at $7.5 \mathrm{MPa}$.

\subsubsection{Wall friction coefficient}

The top wall friction coefficient plays an essential role in the tests, having an enormous effect on the magnitude of peak shear stress. There is no definite formula to obtain a certain figure for friction, but only by trial and error can the optimal friction coefficient be obtained. Figure.9 generally shows that there is a positive correlation between the top wall friction coefficient and peak shear stress. Among three top wall friction coefficients, the simulation results with the interparticle friction coefficient $=0.65$ fit the physical test results.

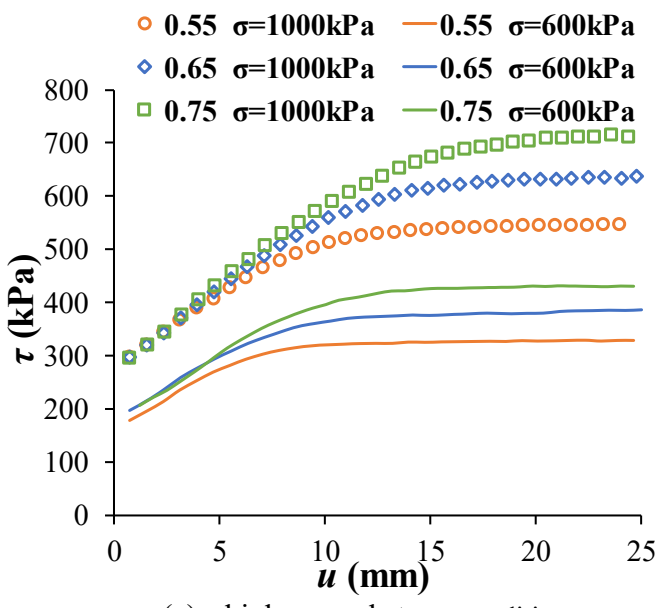

(a) high normal stress conditions 


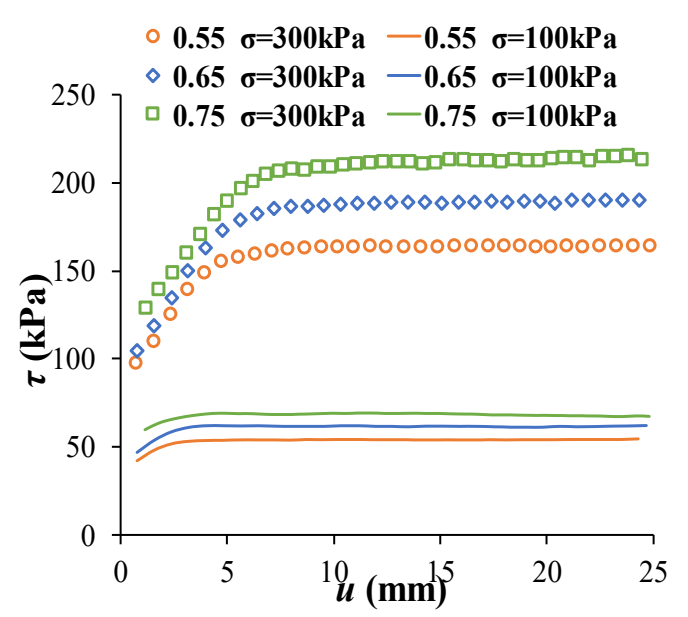

(b) low normal stress conditions

Figure 9. Shear displacement $(u)$ versus shear stress $(\tau)$ with various top wall friction coefficients.

Figures.10 and 11 show a detailed linear relationship between the top wall friction coefficient and the peak shear stress and between the normal stress and the peak shear stress respectively. Basing on these data in Figure.9 and the linear relationships presented in Figs. 10 and 11, the following linear Equation (3) can be derived:

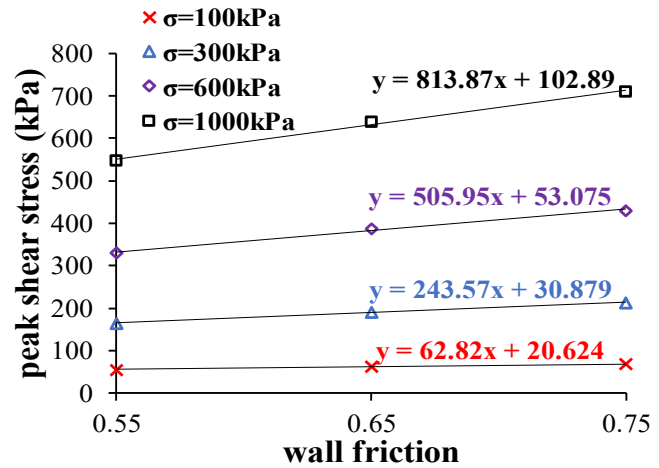

Figure 10. Linear relationship between the top wall friction coefficient and the peak shear stress $(\tau)$ in four normal stress conditions.

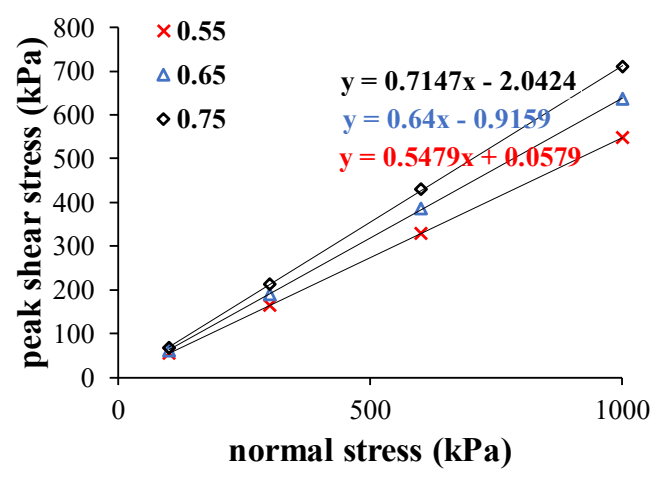

Figure 11. Linear relationship between the normal stress $(\sigma)$ and the peak shear stress $(\tau)$ with three top wall friction coefficients.

$\tau_{\text {peak }}=406.553 \times f_{\text {wall }}+0.634 \times \sigma-265.226$

$\left(\mathrm{R}^{2}=0.9862\right)$ where $\tau_{\text {peak }}$ is the peak shear stress, $f_{\text {wall }}$ is the friction coefficient of the top wall and $\sigma$ is the normal stress.

\subsubsection{Interparticle friction coefficient}

Figure.12 indicates that the interparticle friction influences the peak shear stress, which would grow with the increase of the interparticle friction coefficient. However, the simulation results with interparticle friction coefficients

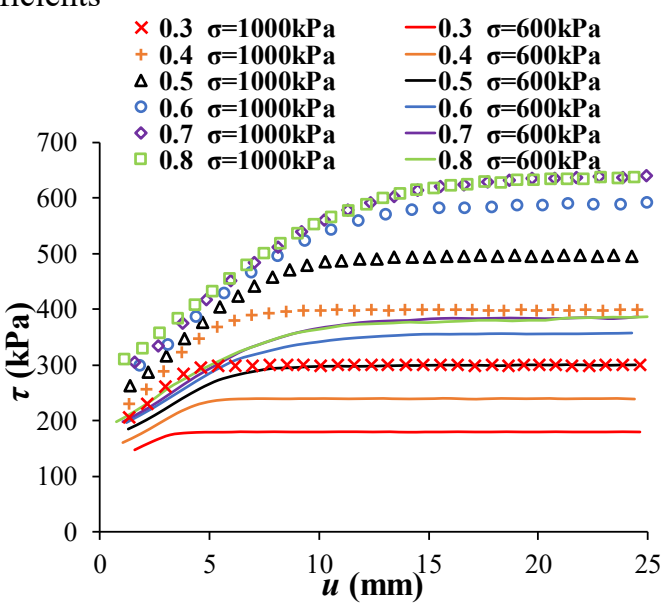

(a) high normal stress conditions

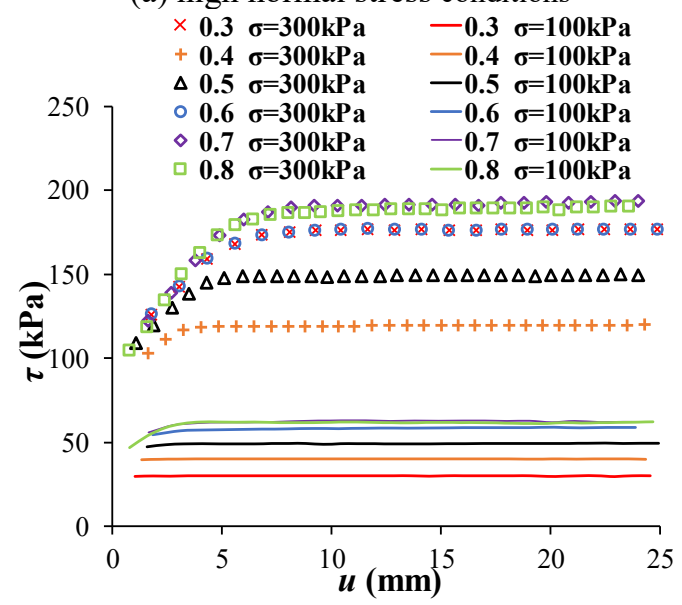

(b) low normal stress conditions

Figure 12. Shear displacement $(u)$ versus shear stress $(\tau)$ with various interparticle friction coefficients.

$=0.7$ and 0.8 are totally the same because of the 'inheritance property' in $\mathrm{PFC}^{3 \mathrm{D}}$, which determines that the deterministic value of the interface is the minimum value between the the top wall friction coefficient and the interparticle friction coefficient. 


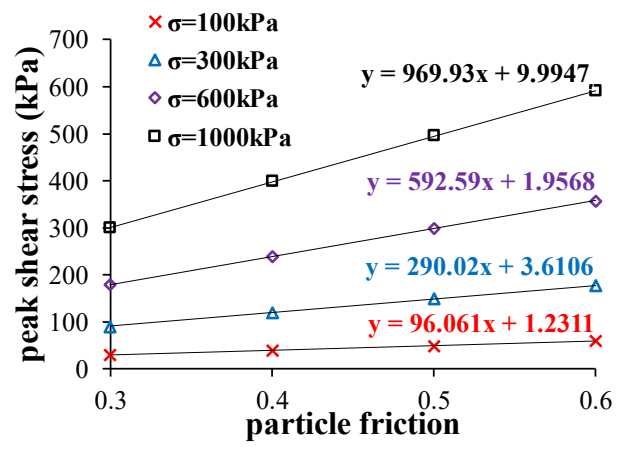

Figure 13. Linear relationship between the interparticle friction coefficient and the peak shear stress $(\tau)$ in four normal stress conditions.

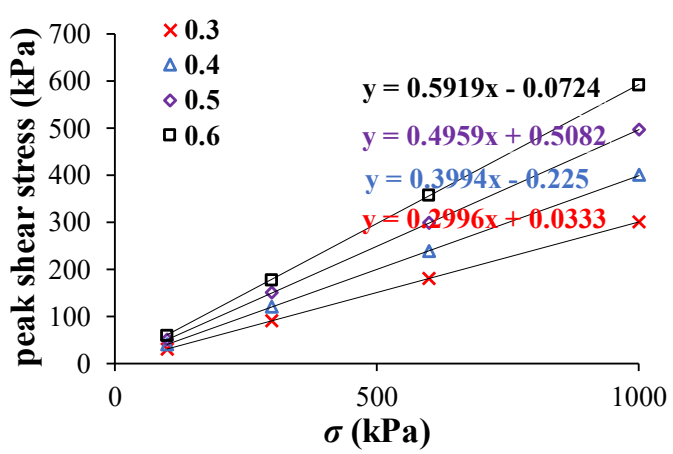

Figure 14. Linear relationship between the normal stress $(\sigma)$ and the peak shear stress $(\tau)$ with four interparticle friction coefficients

Figures.13 and 14 illustrate a detailed linear relationship between the interparticle friction coefficient and the peak shear stress and between the normal stress and the peak shear stress respectively. Basing on these data in Figure. 12 and the linear relationships presented in Figs.13 and 14, the linear Equation (4) is deduced as follows:

$\tau_{\text {peak }}=487.150 \times f_{\text {par }}+0.447 \times \sigma-219.157$

$\left(\mathrm{R}^{2}=0.9423\right)$

where $f_{\text {par }}$ is the interparticle friction coefficient.

\section{Numerical simulation results and analysis}

\subsection{Macroscopic response in the simulation}

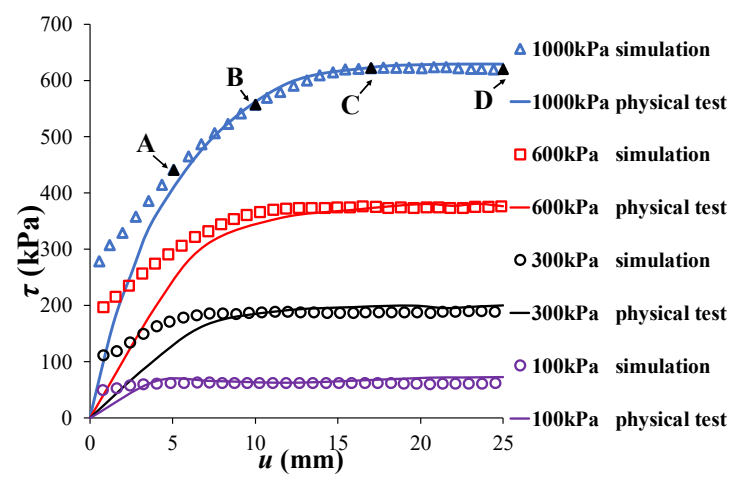

Figure 15. Numerical simulation results and physical test results.

The reason why the shear stresses in the simulation do not grow from zero (unlike physical test results) is that before moving the top wall horizontally, the numerical specimen was applied to a specific value of normal stress and in the process of normal stresses preloading, initial shear stress had thus been generated and would not disappear when the top wall began to move.

Figure. 15 exactly displays that the simulation results fit the physical test results, meaning that these mesoscopic parameters (shown in Table.1) are appropriate in the simulation of the interface test.

As can be seen in Figure.16, the specimen shows an obvious characteristic-dilatancy. At first the volumetric strain $\left(\varepsilon_{v}\right)$ increases slightly, and then after $2.5 \mathrm{~mm}$ displacement of the top wall, the value $\varepsilon_{v}$ begins to decrease remarkably. Until the shear displacement reaches $16 \mathrm{~mm}$, the dropping rate of volumetric strain $\varepsilon_{v}$ starts to slow down and after $22.5 \mathrm{~mm}$ shear displacement, there is almost no change in $\varepsilon_{v}$.

The initial porosity of the specimen is 0.4 . While in the process of applying the inital normal stress, the specimen becomes denser. So the porosity before the shear displacement of the top wall is 0.3866 (Figure.17). The increasing rate of the porosity gradually becomes larger and after $6 \mathrm{~mm}$ shear displacement, the rate becomes stable. Until $16 \mathrm{~mm}$ shear displacement, the incrasing rate of porosity gradually decrease to zero and porosity becomes constant after anout $20 \mathrm{~mm}$ shear displacement of the the top wall. The trend of the porosity is similar to that of volumetric strain $\left(\varepsilon_{v}\right)$ to some extent. 


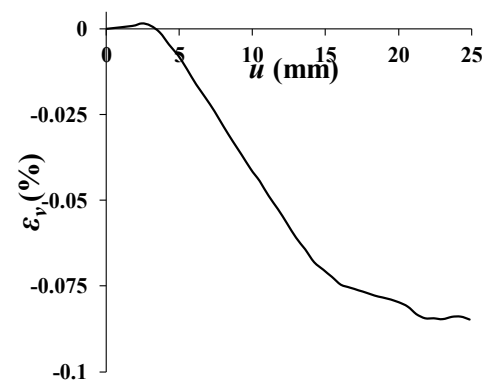

Figure 16. Volumetric strain $\left(\varepsilon_{v}\right)$ versus shear displacement $(u)$ in $1000 \mathrm{kPa}$ normal stress condition (the compression is taken as positive).

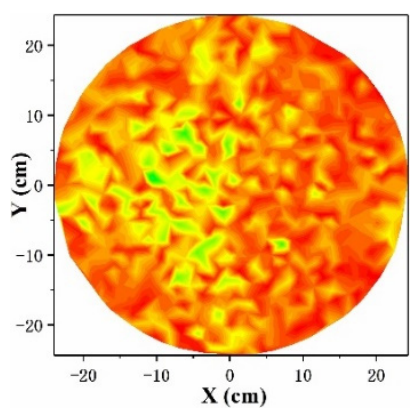

(a)

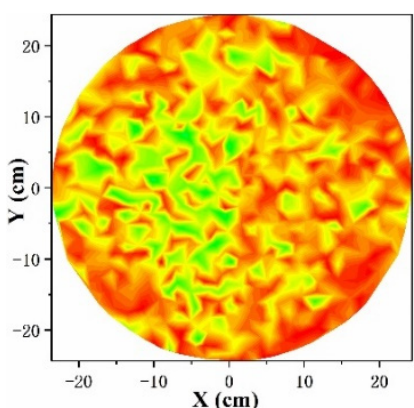

(b)

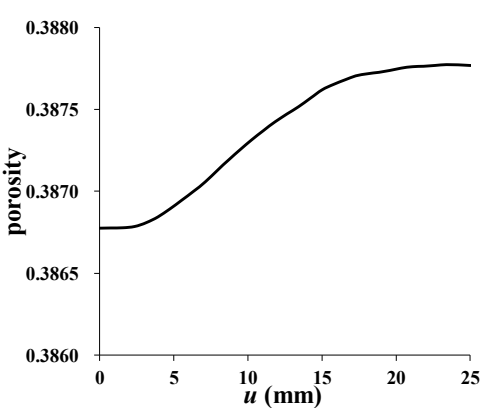

Figure 17. Porosity of the specimen versus shear displacement $(u)$ in $1000 \mathrm{kPa}$ normal stre ss condition

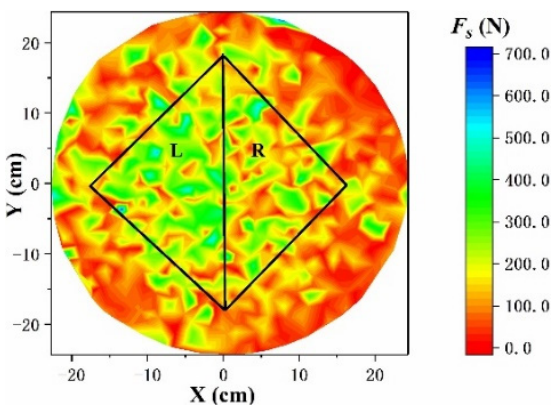

(c)

Figure 18. Interface shear force fields at three stages (a) A point; (b) B point; (c) D point. (these points are shown in Figure.15)

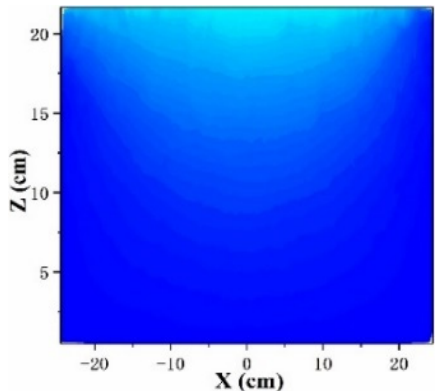

(a)

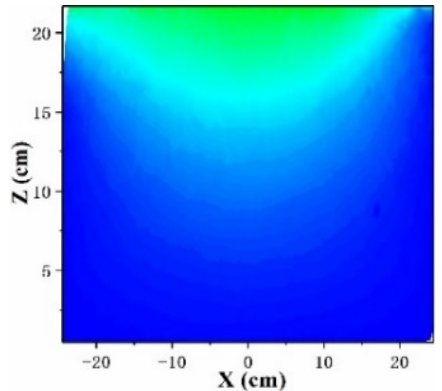

(b)

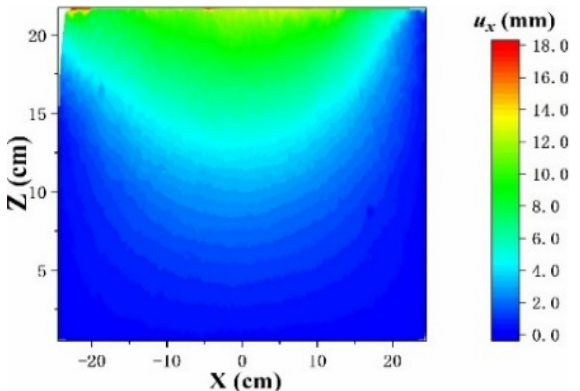

(c)

Figure 19. Particle shear displacement field of A-A section at three stages (a) A point; (b) B point; (c) D point

In general, the shear forces, as can be seen in Figure. 18 on the interface consistently increase. The shear forces in the $\mathrm{L}$ area is growing faster than that in $\mathrm{R}$ area, while the shear forces around the interface do not change a lot, only a few parts of which increase. According to Newton's second law, $F=m a$. In the PFC software, the acceleration is expressed in the displacement in the unit time step. The shear displacements the particles in the center are larger, while those around the are relatively much smaller. (Figure.19). This is why the shear forces in the center are larger than those around the interface. Also, shear forces on the area with $\mathrm{x}$ ranging from $-25 \mathrm{~cm}$ to $15 \mathrm{~cm}$ are larger than those on the area with $\mathrm{x}$ ranging from $15 \mathrm{~cm}$ to $25 \mathrm{~cm}$ because the boundary restricts shear displacements of particles on the latter area and particles on the former area have larger shear displacements than those on the latter one.

\subsection{Evolution on the mesoscopic level}

The green area continuously deepens from Figure.19(a) to Figure.19(c), signifying that the shear displacements of particles in the specimen consistently increase and the shear degree correspondingly grows with the horizontal movement of the top wall. From a single graph, especially from Figure.19(b) or 19(c), shear displacements of particles continuously decrease radially from the center of the top, namly $(0,22.5)$. The depth of green area in Figure.19(c) is nearly up to $12.5 \mathrm{~cm}$, so the influence of the top wall moving horizontally on the particle shear displacement field reach half the height of the specimen.

However, from the perspective of energy dissipation, the influence of the moving top wall moving mainly concentrates on the interface and the area $2.5 \mathrm{~cm}$ below. In this paper, the energy dissipation is only slip energy, which is an energy dissipated by frictional slip. The reason why it does not include the damping energy is that the accurate determination of normal and shear critical damping ratios is unsettled and needs to be discussed. At A point, only two small parts on the left side of the center of the interface $(0,22.5)$ and on the right corner have energy dissipation. When it comes to the $\mathrm{B}$ point, a small part on the right side of and adjacent to the center has the 
slip energy disspation as well, but the value of it is relatively smaller than two

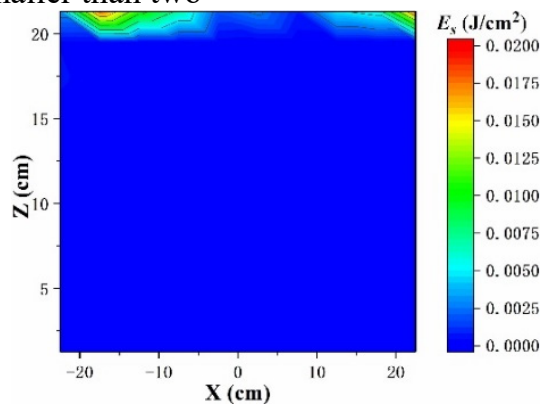

(a)

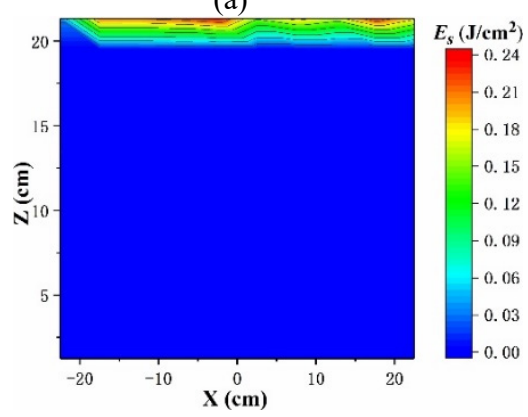

(c)

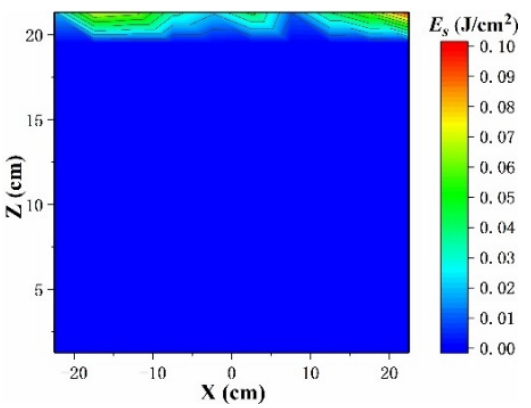

(b)

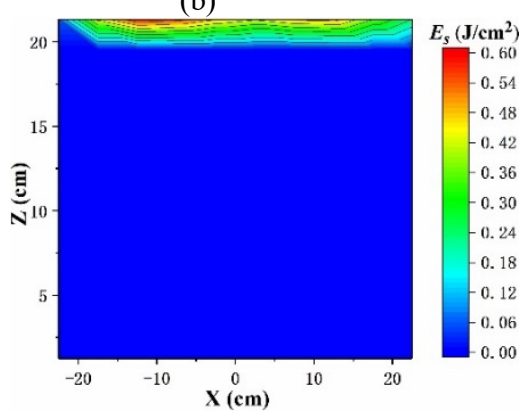

(d)

Fig 20. Incremental energy dissipation density distribution of A-A section at four stages (a) A point; (b) B point; (c) c point; (d) D point.

other parts mentioned before.From the $\mathrm{C}$ point, almost all parts of the interface and the area $2.5 \mathrm{~cm}$ below have energy dissipation. The density distribution of the incrmental energy dissipation decreases from the interface to the height at $20 \mathrm{~cm}$. In general, the increasing rate of the energy dissipation are getting faster and faster. The biggest incmrental energy dissipation at $\mathrm{D}$ point is about 30 times that at $\mathrm{A}$ point. In addition, the range of the incremental energy dissipation density distribution is like a shear band.

\section{Conclusion}

(1) Cohesion strength and tensile strength determine whether there is strain softening or strain hardening.

(2) Cohesion strength, tensile strength and the top wall and interparticle friction coefficients all remarkably affect the peak shear stress. The determination of these parameters is of great importance to whether the numerical simulations can reproduce the macroscopic behaviours in the physical tests.

(3) Dilatancy occurs during the shearing process and the porosity of the specimen increases generally. Also, shear stresses of the interface, shear displacements of particles and energy dissipation all increase during the shearing process.

(4) Shear forces mainly concentrate on the central part of the interface and incremental energy dissipation concentrates on the top $2.5 \mathrm{~cm}$ area.

\section{References}

1. G.W. Clough, J.M. Duncan. Finite element analysis of retaining wall behaviour. J Soil Mech Found Div, ASCE, 97(12):1657-73. (1971)

2. C.S Desai, Y. Ma. Modelling of joints and interfaces using the disturbed-state concept. Int. J. of Numerical \& Analytical Methods in Geomechanics, 16:623-653. (1992)

3. C.S Desai, E.C Drumm. Cyclic testing and modeling of interfaces. J. Geotech Eng, 111(6):793-815. (1985)

4. G. Zhang, J.M Zhang. Numerical modelling of soilstructure interface of a concrete-faced rockfill dam. Comput Geotech, 36:762-772. (2009)

5. P.A Cundall. A discrete numerical model for granular assemblies. Géotechnique, 29:47-65. (1979)

6. X.H Gu, M.S Huang, J.S Qian. Discrete element modeling of shear band in granular materials. Theor Appl Fract Mec, 72:37-49. (2014)

7. Q Zhang, W.Y Xu, Q.Y Liu. Numerical investigations on mechanical characteristics and failure mechanism of outwash deposits based on random meso-structures using discrete element method. J Cent South Univ, 24 :2894-2905. ( 2017)

8. T.G Sitharam, S. V. Dinesh. Numerical simulation of liquefaction behaviour of granular materials using Discrete Element Method. Proc. Indian Acad, 112:479-484. (2003)

9. Itasca Consulting Group Inc. PFC 5.0 Documentation. (2017) 